\title{
On the mechanism of adhesion in biological systems
}

\author{
B. N. J. Persson ${ }^{a)}$ \\ IFF, FZ-Jülich, 52425 Jülich, Germany
}

(Received 7 November 2002; accepted 28 January 2003)

\begin{abstract}
I study adhesion relevant to biological systems, e.g., flies, crickets and lizards, where the adhesive microstructures consist of arrays of thin fibers. The effective elastic modulus of the fiber arrays can be very small which is of fundamental importance for adhesion on smooth and rough substrates. I study how the adhesion depend on the substrate roughness amplitude and apply the theoretical results to lizards. (C) 2003 American Institute of Physics. [DOI: 10.1063/1.1562192]
\end{abstract}

\section{INTRODUCTION}

How can a fly or a cricket walk on a glass window, or a lizard move on a stone or concrete wall? These fundamental questions have interested scientists for many years, and recently very important work has been performed in order to gain a deeper insight into these questions. ${ }^{1}$ In this paper, I focus mainly on dry adhesion which seems to be relevant for lizards, ${ }^{2}$ and I present a simple model study of the influence of surface roughness on the adhesion between a lizard toe and a smooth or rough hard substrate.

The adhesive microstructures of lizard is the results of perhaps millions of year of development driven by the principle of natural selection. Hence one may expect the adhesive structures to be highly optimized and it is clear that a good understanding of the construction and function of the adhesive structures may lead to new improved man-made adhesives.

The breaking of adhesive bonds between macroscopic bodies (including biological systems) usually occur by the propagation of a crack from the periphery of the contact area towards the center. The process of crack propagation depends on the elastic modulus $E$ of the solids, and on the energy per unit area, $\gamma_{\text {eff }}$, to propagate the crack. The latter is a dynamical quantity which depends on the speed of the crack edge. In many cases, including the systems studied below, $\gamma_{\text {eff }} \gg \Delta \gamma$, where $\Delta \gamma=\gamma_{1}+\gamma_{2}-\gamma_{12}$ is the change in the surface free energy when the two solids make contact. Note that the magnitude of $\Delta \gamma$ reflect the strength of the interfacial bonding between the two solids, and may be written as $\Delta \gamma=n^{*} f^{*} b^{*}$, where $b^{*}$ is of order the distance necessary to break an interfacial bond, $f^{*}$ is the typical force necessary to break a bond, and $n^{*}$ is the number of bonds per unit area. In general, $b^{*}$ will be of order a few Angstrom. The condition $\gamma_{\text {eff }} \gg \Delta \gamma$ is obeyed if the distance $b$ the solid walls must be separate in order to break the interfacial bonds is much larger than atomic distance $b^{*}$. This is the case in many important applications. For example, for polymers in contact with a substrate, the bond breaking may involve the pull-out of polymer chains, ${ }^{3}$ or the formation of bundles of polymer chains (crazes) connecting the two solid walls ${ }^{4}$ (see Fig. 1). In this case $b$ is typically very large, e.g., for adhe-

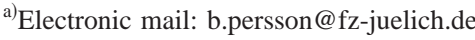

sive tape $b$ may be of order a few mm. In biological applications another mechanism leads to a similar effect: The surface of many biological systems are covered by many thin curved fibers (hair), which can bind to a substrate (see Fig. 2). During pull-off the fibers at the crack edge straighten out, and may elongate many micrometers before the force in the fiber is high enough to break the bond to the substrate (this effect has not been studied experimentally, but must occur). If $k$ denotes the effective spring constant of the fiber (see below), and if the fiber-substrate bond breaks when the fiber force equals $f$, then (for a smooth substrate) $\gamma_{\mathrm{eff}}=n k b^{2} / 2$, where $n$ is the number of fibers per unit area and where the displacement $b$ is determined by $k b=f$. Since the spring constant $k$ associated with a long (curved) fiber is very small, the displacement $b$ may be very large (e.g., 10 micrometers) leading to a very large effective surface energy $\gamma_{\text {eff }}$. Thus we may loosely state that strong adhesion results from "long bonds" rather than from "strong bonds." This is perhaps the most important general statement related to adhesion, and it forms the basic construction-principle for both biological and man-made adhesive systems.

It is important to note that most natural surfaces are rather inert; any (unsaturated) high-energy bonds which may have occurred on a freshly formed surface (e.g., a surface formed by cleavage), would have been rapidly passivated by the reaction with molecules from the atmosphere. Further- (a)

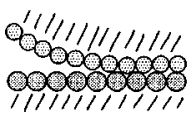

(b)

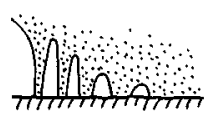

(c)

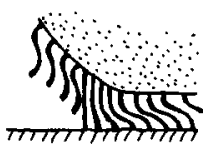

FIG. 1. Three different cases illustrating the breaking of an adhesion junction via crack propagation. In all cases the substrate is assumed to be rigid. (a) Crack propagation at an ideal brittle interface between two different materials. The crack propagates by stretching and breaking the atomic bonds at the crack tip and $\gamma_{\text {eff }} \approx \Delta \gamma$. (b) Contact between a hard solid substrate and a soft (weakly cross-linked) polymer. The crack propagate by pulling out polymer strings leading to an effective long-range interaction between the walls, and to a large crack propagation energy, $\gamma_{\mathrm{eff}} \gg \Delta \gamma$. (c) Contact between a hard solid substrate and a softer solid covered by thin curved elastic fibers making adhesive contact to the substrate. Here crack propagation occurs via stretching of the fibers before the fiber-substrate bonds break. For long fibers this results in an effective long-ranged interaction between the walls, and $\gamma_{\mathrm{eff}} \gg \Delta \gamma$. 


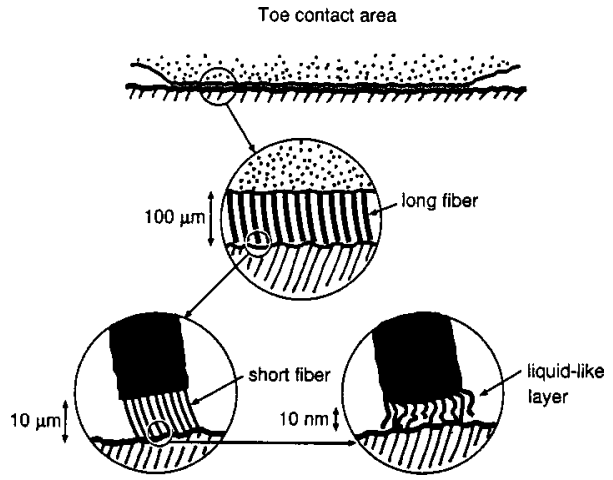

FIG. 2. The footprint of a lizard toe with a smooth substrate. On the longest length scale the contact occurs between the lizard toe skin and the substrate over an area $\sim 10 \mathrm{~mm}^{2}$. The skin is covered by long fibers or hair (setae) (length $\approx 200 \mu \mathrm{m}$ and radius $\approx 3 \mu \mathrm{m}$ ), which in turn have shorter fibers (spatula) (about 1000 per setae, of length $\approx 20 \mu \mathrm{m}$, and radius $\approx 0.1 \mu \mathrm{m}$ ) at the end. The short fibers end with a thin (10-100 nm thick) leaflike sheets (not shown). In addition, the top of each short fiber has, most likely, a thin high mobility, liquidlike layer (thickness unknown but probably of order 10 $\mathrm{nm}$ ) which allows the fiber to make atomic contact with surfaces with atomic scale roughness.

more, most real surfaces are covered by thin inert contamination films, e.g., nanometer thick organic layers. Thus, it is usually not possible to form strong covalent or ionic bonds between biological surfaces and most "natural" surfaces exposed to the normal atmosphere. This fact may even be advantageous since strong bonds to the substrate may lead to large wear during lateral movement involving the formation and breaking of adhesive bonds. Thus, it is clear that in most cases the van der Waals interaction (which occurs between all bodies) will be the most important adhesive force. In fact, even in the situation where a thin (high viscosity) liquid layer is introduced at the interface between the biological surface and the substrate, its main role may be to smoothen the rough substrate surface, and thus effectively increasing the contact area between the two solids, resulting in a strengthening of the adhesive bond.

The adhesion between an elastic solid (rubber) and a hard rough substrate has been studied in a classic work by Fuller and Tabor ${ }^{5}$ and also by Briggs and Briscoe. ${ }^{6}$ They found that already a relative small surface roughness may result in negligible adhesion. Thus a surface roughness of $\sim 10 \mu \mathrm{m}$ (root-mean-square amplitude) may completely remove the adhesion between a very soft rubber ball (elastic modulus $E \approx 0.06 \mathrm{MPa}$ ) and a hard rough substrate. The outermost layer of the skin on the toe of a lizard is made from a relatively stiff material (keratin, with the elastic modulus $E$ $\approx 4 \mathrm{GPa}$ ), which has an elastic modulus $\sim 10^{5}$ times higher than that of very soft rubber. Thus, without the soft compliant fiber array system discussed above, no detectable adhesion would occur between a lizard toe and a rough substrate, such as a stone or a concrete wall.

Dry adhesion between an elastic solid and a hard rough substrate results from the competition between two energy terms, namely the molecular binding energy (or adhesion energy) $E_{\text {ad }}$ originating from the contact regions between the solids, and the elastic deformation energy $E_{\mathrm{el}}$ stored at the interface as a result of the deformations necessary in order to bring the solids into molecular contact at the interface. If the surface roughness is "large enough" the elastic energy and the molecular binding energy terms will be of nearly equal magnitude but of opposite sign, so that the total interfacial energy will nearly vanish, $E_{\mathrm{ad}}+E_{\mathrm{el}} \approx 0$. If during pull-off the elastic energy stored in the deformation field at the interface is given back to the system (which may require that the pulloff is very slow so that we can neglect inelastic events), no net force would be necessary in order to break the bond between the two solids and the adhesion would vanish. That is, if the total interfacial energy vanishes, the elastic energy stored at the interface is just large enough to break the interfacial bonds between the solids. However, in the case of fiber adhesion it turns out to be impossible to neglect inelastic events, and strong adhesion is possible also when $E_{\mathrm{el}}+E_{\mathrm{ad}}$ $>0$, see Sec. IV. We note that calculations have shown that even in the case where the total interfacial energy (nearly) vanishes, there is still a finite adhesion-induced increase in the contact area between the solids, ${ }^{7}$ which would manifest itself in, e.g., a sliding friction experiment.

\section{PULL-OFF FORCE}

Irshick et al. ${ }^{8}$ have demonstrated that the feet of a gecko can adhere to a substrate with a force $\sim 10 \mathrm{~N}$ (corresponding to the weight of $1 \mathrm{~kg}$ !). The typical weight of a tokay is approximately $40 \mathrm{~g}$ meaning that only $1 \%$ of the maximum adhering force (assuming 4 gecko feet) is required to support the whole weight of the gecko. This raises the question of why gecko are apparently so over-built. However, we will show below that the adhesion to rough surfaces can be strongly reduced (and even vanish if the root mean square amplitude of the roughness is high enough). Furthermore, sometimes a gecko may need to resist very large (inertia) forces applied over a short duration, e.g., to attach to a solid wall during falling. ${ }^{9,10}$ However, in this case the frictional forces are additionally involved in the attachment.

Autumn et al. ${ }^{2}$ have measured the force to break the bond between a single setae and a flat substrate. They observed a maximum pull-off force of order $\sim 200 \mu \mathrm{N}$. If all 6.5 million setae of a gecko would have to be broken simultaneously, the pull-off force would be of order $1300 \mathrm{~N}$ (see Refs. 2 and 9), i.e., about a factor 30 larger than the maximal observed gecko pull-off force. However, the bond between two macroscopic solids is (nearly) never broken uniformly over the contact area, but rather occur by the propagation of an interfacial crack. During pull-off, at the crack edge the local stress is strongly increased above the average tensile stress in the contact area. We believe that this is the origin of the factor of 30 difference in the calculated (based on the assumption that all the setae-substrate bonds break simultaneously) and the observed pull-off force (see below).

Interfacial cracks can nucleate either at defects at the interface (e.g., where the two surfaces are separated by a small particle, e.g., a sand particle) or, more likely, it will start at the periphery of the contact area and propagate towards the center during pull-off. The pull-off force depends in general on the shape of the bodies, on the elastic modulus $E$, and on the crack propagation energy (per unit area) $\gamma_{\mathrm{eff}}$. If we assume a hard and nominally flat substrate in contact 
(a)

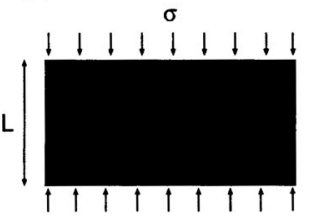

(b)

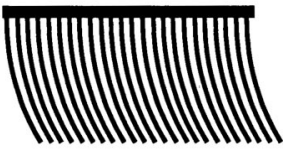

FIG. 3. (a) When an elastic block of thickness $L$ is exposed to the surface stress $\sigma$ it will deform a distance $u$ so that the strain $u / L=\sigma / E$, where $E$ is the elastic modulus. (b) If the solid slab in (a) is replaced by a dense array of thin curved fibers, the effective elastic modulus $E^{*}$ is strongly decreased, e.g., by a factor $\sim 10^{4}$ for the setae array on the toe of a lizard.

with a soft elastic body (elastic modulus $E$ ) with the radius of curvature $R$, the pull-off force is independent of $E$ and given by the JKR theory: ${ }^{11}$

$$
F_{\mathrm{ad}}=\frac{3}{2} \pi R \gamma_{\mathrm{eff}} .
$$

Since the contact mechanical properties of a gecko toe-pad is likely to be visco-elastic and nonlinear (as has been observed for the human finger-pad ${ }^{12}$ ), Eq. (1) is only of very approximative validity in the present application. If we assume that the local radius of curvature $R \approx 1 \mathrm{~cm}$ (which is rather large, but the gecko toe-pad is rather flat) and if we use the observed (maximal) pull-off force $F_{\text {ad }}=2 \mathrm{~N}$, we get $\gamma_{\text {eff }} \approx 40$ $\mathrm{J} / \mathrm{m}^{2}$ or $2 \mathrm{eV} / \AA^{2}$. This is about 1000 larger than the change in the interfacial energy $\Delta \gamma$ due to the van der Waals interaction, which is typically of order a few $\mathrm{meV} / \AA^{2}$. This large crack propagation energy is typical for pressure sensitive adhesives, which consist of thin polymer layers which, during pull-off, undergoes stringing at the crack edge as illustrated in Fig. 1(b). However, in the present case the mechanism for the enhancement of $\gamma_{\text {eff }}$ above $\Delta \gamma$ is different, and related to the stretching of curved fibers as illustrated in Fig. 1(c) and discussed in Sec. IV.

We note that the assumption that not all the interfacial bonds break simultaneously is central to adhesion in general. If all the atomic bonds would break simultaneously at the interface, the pull-off stress would be of order $\Delta \gamma / b^{*}$, where $b^{*}$ is the bond length. Since $b^{*}$ is of order a few Angstrom and $\Delta \gamma$ of order a few $m e V / \AA^{2}$, we get the ideal pull-off stress $\approx 200 \mathrm{MPa}$ which is 400 times higher than the observed pull-off stress $(0.5 \mathrm{MPa})$.

\section{EFFECTIVE ELASTICITY OF FIBER-ARRAY SYSTEMS}

The great innovation made by nature in the context of biological adhesive systems (e.g., in insects or lizards) is the discovery that arrays of curved fibers may be elastically extremely soft, and hence can deform and make contact everywhere at the interface even when the substrate is very rough (but not too rough; see below). Let us compare the elastic modulus of a solid slab [see Fig. 3(a)] with the effective elastic modulus of a fibrous material made from the same material; see Fig. 3(b). If we apply a stress $\sigma$ to the solid slab in (a) then the strain $u / L$ is given by $\sigma=E u / L$ where $E$ is the elastic modulus. On the other hand, if we apply a force $F$ to a fiber (radius $R$ ) (see Fig. 4) this will induce a displacement

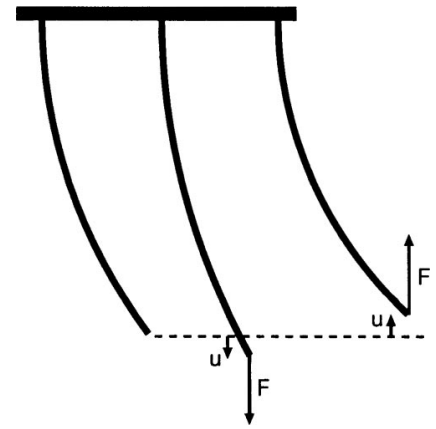

FIG. 4. When a curved elastic fiber is exposed to a force $F$ it will displace a distance $u=F / k$ which is proportional to $F$ for small $F$, where the spring constant $k$ is given by the theory of elastic beams.

$u$ given by $F=k u$ where the effective spring constant $k$ $=C E R^{4} / L^{3}$, where $C$ is a number which depends on the shape of the fiber but which typically is of order 10 (see Appendix A). If there are $N$ fibers on the area $A$ then the normal stress $\sigma=N F / A=N k u / A \equiv E^{*} u / L$, where the effective modulus,

$$
E^{*}=\frac{N k L}{A}=C E \frac{N R^{2}}{A}\left(\frac{R}{L}\right)^{2} .
$$

For the setae array we have $R / L \approx 0.02$. In the gecko toe-pad occur $\sim 10^{4}$ setae per $\mathrm{mm}^{2}$ contact area, giving $N R^{2} / A$ $\approx 0.02$ so that $E^{*} \approx 10^{-4} E$. Thus the replacement of the solid block in Fig. 3(a) with an array of fibers (of the same material), reduce the effective elastic modulus from $E \approx 4$ $\mathrm{GPa}$ to $E^{*}=0.4 \mathrm{MPa}$, which is similar to that of relative soft (sticky) rubber. This is the fundamental mechanism by which many biological objects generate "sticky" surfaces.

Using micro-indentation experiments, Scherge and Gorb $^{1}$ have measured the effective elastic properties of the fiber-array layer of the pad of the great green bush cricket. For a small indentation they observed $E^{*} \approx 10 \mathrm{kPa}$. The fiber array consists of $L \approx 100 \mu \mathrm{m}$ long fibers with the radius $R$ $\approx 0.5 \mu \mathrm{m}$. The separation between the fibers is about $8 \mu \mathrm{m}$ and assuming a hexagonal arrangement gives $N R^{2} / A$ $\approx 0.005$. The fibers are made from cuticle, which is a composite material consisting of chitin fibers and protein matrix, with an elastic modulus in the range ${ }^{13} E=0.3-13 \mathrm{GPa}$. Thus we get $E^{*}$ (theory) $\approx 0.4-16 \mathrm{kPa}$ which is consistent with the experimental observation.

\section{FIBER-ARRAY ADHESION ON HARD ROUGH SURFACES}

If $\epsilon_{0}$ denote the fiber-substrate binding energy, then the total energy equals

$$
U=\sum_{i}\left[\frac{1}{2} k\left(z_{0}-h_{i}-L\right)^{2}-\epsilon_{0}\right],
$$

where the sum is over all the attached fibers. Here $L$ is the natural length of the fiber, $h_{i}$ the substrate surface roughness height at fiber $i$ and $z_{0}$ the separation between the base of the fiber array and the substrate (see Fig. 5). Assume that the system is "prepared" by squeezing the upper solid towards the substrate until $z=z_{0}^{0}$. In this case fiber $i$ will make con- 


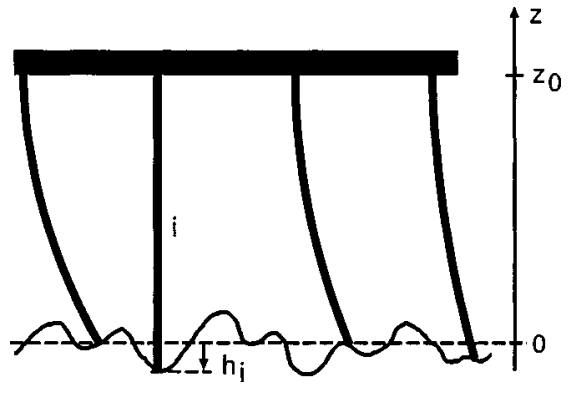

FIG. 5. Fiber array in contact with a rough substrate. The dashed line describes the average surface height, from which $h(\mathbf{x})$ is measured so that $\langle h\rangle=0$.

tact with the substrate if $z_{0}^{0}-h_{i}<L$. Let $P(h)$ be the surface height probability distribution where the origin of $h$ is chosen so that $\langle h\rangle=0$. Consider now a pull-off experiment where $z_{0}>z_{0}^{0}$. We can write the total energy as

$$
\begin{aligned}
U= & N_{0} \int_{h^{*}}^{z_{0}} d h P(h)\left[\frac{1}{2} k\left(z_{0}-h-L\right)^{2}-\epsilon_{0}\right] \\
& \times \theta\left(h-z_{0}^{0}+L\right),
\end{aligned}
$$

where $N_{0}$ is the total number of fibers. The $\theta$-function in (2) describes the fibers which made initial contact, and $h^{*}$ is the smallest $h$ for which fiber-substrate contact can occur. We can relate $h^{*}$ to the force $f$ necessary in order to break a fiber-substrate bond. The elastic force in an attached fiber is

$$
-\frac{\partial U}{\partial h_{i}}=k\left(z_{0}-h_{i}-L\right),
$$

and when this equals $f$ the bond breaks. This gives

$$
h^{*}=z_{0}-L-f / k \text {. }
$$

In what follows we will assume that $P(h)$ is a Gaussian:

$$
P(h)=\left(\frac{1}{2 \pi \sigma^{2}}\right)^{1 / 2} \exp \left(-\frac{h^{2}}{2 \sigma^{2}}\right) .
$$

The root mean square $(\mathrm{rms})$ roughness amplitude $\sigma$ does not refer to the total surface area of the substrate, but only to a small region, which, for most biological applications, has a diameter of order $D \approx 1000 \mu \mathrm{m}$ (see below). Substituting (3) and (4) in (2), and writing $x=\left(h-z_{0}+L\right) / \sigma$ gives

$$
\begin{aligned}
U= & \frac{N_{0}}{(2 \pi)^{1 / 2}} \int_{-f / k \sigma}^{L / \sigma} d x \exp \left(-\frac{1}{2}\left[x+\frac{z_{0}-L}{\sigma}\right]^{2}\right) \\
& \times\left[\frac{1}{2} k \sigma^{2} x^{2}-\epsilon_{0}\right] \theta\left[x-\left(z_{0}^{0}-z_{0}\right) / \sigma\right] .
\end{aligned}
$$

If we define the adhesion length parameter $\sigma_{0}=f / k$ and the elastic energy,

$$
\epsilon_{\mathrm{el}}=\frac{1}{2} k \sigma_{0}^{2}
$$

and if we assume that initially all the fibers make contact with the substrate, then (5) can be written as

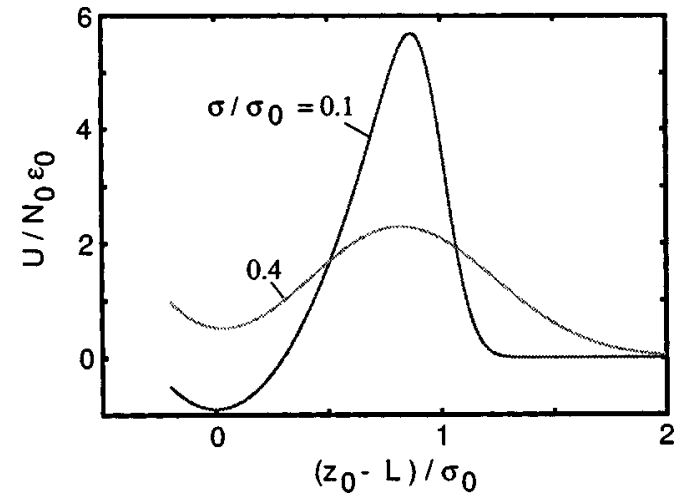

FIG. 6. The energy $U$ is shown as a function of $y=\left(z_{0}-L\right) / \sigma_{0}$ for $\epsilon_{\mathrm{el}} / \epsilon_{0}$ $=10$, and for $\sigma / \sigma_{0}=0.1$ and 0.4. Note that for $\sigma / \sigma_{0}=0.4$ the energy at the minima $\left[\right.$ for $\left(z_{0}-L\right) / \sigma_{0} \approx 0$ ] is positive and hence larger than the energy $U=0$ of the noncontacting state.

$$
\begin{aligned}
U= & \frac{N_{0}}{(2 \pi)^{1 / 2}} \int_{-\sigma_{0} / \sigma}^{L / \sigma} d x \exp \left(-\frac{1}{2}\left[x+\frac{z_{0}-L}{\sigma}\right]^{2}\right) \\
& \times\left[\epsilon_{\mathrm{el}}\left(\frac{\sigma}{\sigma_{0}}\right)^{2} x^{2}-\epsilon_{0}\right] .
\end{aligned}
$$

In (7) we can in most applications replace the upper limit $L / \sigma$ in the integral with infinity. The energy $U$ (in units of $\left.N \epsilon_{0}\right)$ is shown as a function of $y=\left(z_{0}-L\right) / \sigma_{0}$ in Fig. 6 for $\epsilon_{\mathrm{el}} / \epsilon_{0}=10$, and for $\sigma / \sigma_{0}=0.1$ and 0.4 . Note that for $\sigma / \sigma_{0}$ $=0.4$ the energy at the minima for $\left(z_{0}-L\right) / \sigma_{0} \approx 0$ is positive and hence larger than the energy $U=0$ of the noncontact state. Thus, in this case the attached state is, even in the absence of a pulling force, metastable. Thus, fiber adhesion is an example where strong adhesion is possible even when $U=E_{\text {el }}+E_{\text {ad }}>0$; this is possible only because of strong inelastic events during pull-off. The barrier separating the local minima from the detached fiber state is, in a typical application (see Sec. V), extremely high, of order $\epsilon_{\mathrm{el}} \sim 10^{10} \mathrm{eV}$, and thermally activated jumps over the barrier have a negligible probability, even when the system is close to the top of the barrier during a pull-off experiment. This does not imply that temperature effects are negligible since the force $f$ to break a fiber-substrate bond, will in general depend on temperature. Since we can neglect thermally activated jump over the barrier in Fig. 6, a finite energy $W$, given by the barrier height between the metastable state and the detached state, is necessary in order to break the contact.

When $\epsilon_{\mathrm{el}} / \epsilon_{0} \gg 1$, which is satisfied in our applications, we can approximate

$$
\begin{aligned}
U= & -N_{0} \epsilon_{\mathrm{el}}\left(\frac{\sigma}{\sigma_{0}}\right)^{2} \frac{1}{(2 \pi)^{1 / 2}} \int_{-\sigma_{0} / \sigma}^{L / \sigma} d x x^{2} \\
& \times \exp \left(-\frac{1}{2}\left[x+\frac{z_{0}-L}{\sigma}\right]^{2}\right) .
\end{aligned}
$$

The work $W$ to break $N_{0}$ fiber-substrate bonds is given by the difference between the maximum of $U(y)$, and the minimum of $U(y)$ in the attached state (where $y \approx 0$; see Fig. 6). We get 


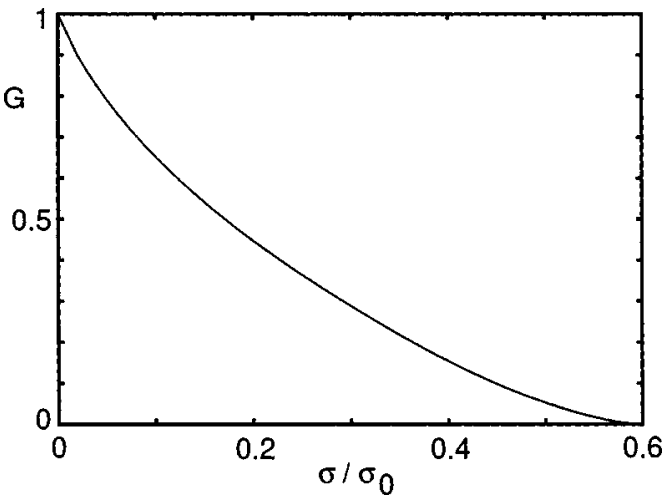

FIG. 7. The function $G\left(\sigma / \sigma_{0}\right)$ is proportional to the work necessary to break the fiber-substrate bonds. See the text for details.

$$
W=N_{0} \epsilon_{\mathrm{el}} G\left(\sigma / \sigma_{0}\right),
$$

where $G$ is shown in Fig. 7. We define the effective surface energy per unit area,

$$
\gamma_{\mathrm{eff}}=\left(N_{0} / A_{0}\right) \epsilon_{\mathrm{el}} G\left(\sigma / \sigma_{0}\right) .
$$

Finally, let us determine the lateral size $D$ of the surface area over which the rms roughness amplitude $\sigma$ should be measured. This is a crucial problem since many real surfaces (e.g., surfaces prepared by fracture) are self affine fractal without any long-distance cut off (or, more accurately, the long-distance cut off is determined by the lateral size of the physical object, which can be very large, e.g., a mountain), which implies that $\sigma$ increases without limit as the surface area over which it is measured increases. To determine the diameter $D$ we note first that the fiber array on the toe-pad is connected to a thin keratin layer (the top layer of the skin) with a thickness of order $d \approx 100 \mu \mathrm{m}$, and with an elastic modulus of order $E \approx 4 \mathrm{GPa}$. Because of its high elastic modulus, the skin is not able to deform and follow the substrate short-wavelength surface roughness; it is for exactly this reason that the toe-pad skin is covered by the fiber array system, which forms a very soft compliant layer. In the calculation presented above we have assumed that the fibers are connected to a rigid skin surface. However, the keratin skin layer will deform, and follow the very long-wavelength surface roughness components. The distance $D$ equals the crossover wavelength, below which only the fiber array system deforms (while the skin or keratin layer is effectively rigid and flat), and above which the skin deform to follow the surface roughness profile. We can determine $D$ as follows: Let us estimate the elastic energy necessary to deform the keratin slab so that it bends into a substrate "cavity" of width $D$ and depth $u$. If we assume that $D \gg d$ (where $d$ is the thickness of the keratin slab), we can use the theory of elasticity for thin plates to estimate the elastic bending energy. We get $E_{\text {plate }}(D) \approx E d^{3} u^{2} / D^{2}$ (see Appendix B), where $E$ is the elastic modulus of the keratin slab, which we assume is (roughly) the same as the elastic modulus of the material from which the fiber array is made. Now, if this energy is larger than the energy $E_{\text {fiber }}(D)$ necessary to deform the fiber array (bound to a flat keratin slab) by a distance $u$ within the area $D \times D$, then the keratin layer will not deform but rather the fiber array will deform. Thus, the cross-over distance $D$ from keratin-plate deformation for surface roughness wavelength $\lambda>D$, to fiber array deformation for $\lambda<D$, is determined by the condition $E_{\text {plate }}(D) \approx E_{\text {fiber }}(D)$. Since the fiber array deformation energy is of order $E_{\text {fiber }} \approx n D^{2} k u^{2}$ we get

$$
E d^{3} / D^{2} \approx n D^{2} k
$$

or

$$
D \approx\left(\frac{E d^{3}}{n k}\right)^{1 / 4}
$$

Using the expression for $k$ derived in Appendix A gives

$$
D \approx d\left(\frac{(L / d)(L / R)^{2}}{n R^{2}}\right)^{1 / 4} .
$$

Since $n R^{2} \approx 0.01, L / d \approx 1$, and $L / R \approx 100$ we get

$$
D \approx 20 d \approx 2000 \mu \mathrm{m}=2 \mathrm{~mm} .
$$

Thus, the condition $D \gg d$ is reasonable well satisfied, and the elasticity theory of thin plates should be a good approximation.

In practice, there are two ways to measure $\sigma$ over the length scale $D$. Either it is measured directly using, e.g., an Atomic Force Microscopy, or else it is deduced from the surface roughness power spectra $C(q)$, which may have been measured over a much larger surface area than $D \times D$. Note that

$$
C(q)=\frac{1}{(2 \pi)^{2}} \int d^{2} x\langle h(\mathbf{x}) h(\mathbf{0})\rangle e^{-i \mathbf{q} \cdot \mathbf{x}}
$$

or

$$
\langle h(\mathbf{x}) h(\mathbf{0})\rangle=\int d^{2} q C(q) e^{i \mathbf{q} \cdot \mathbf{x}},
$$

so that

$$
\left\langle h^{2}\right\rangle=\int d^{2} q C(q) .
$$

The square of the height fluctuation measured over the area $D \times D$ can now be written as

$$
\left\langle h^{2}\right\rangle_{D}=\int_{q>q_{\mathrm{c}}} d^{2} q C(q),
$$

where the cut-off wave vector $q_{\mathrm{c}} \approx 2 \pi / D$. If $\left\langle h^{2}\right\rangle_{D}$ is measured directly, one must measure $h(\mathbf{x})$ relative to a reference plane chosen so that not only $\langle h\rangle_{D}=0$, but also so that $\left\langle h^{2}\right\rangle_{D}$ is minimal [see Fig. 8(a)], which requires using a "tilted" reference plane, where the orientation (tilt angle) depends on the location of the $D \times D$ patch on the substrate surface; see Fig. 8(b).

\section{APPLICATION TO LIZARDS}

Let us estimate $\sigma_{0}$ and $\epsilon_{\mathrm{el}} / \epsilon_{0}$ for the setae of the Tokay gecko. Consider a curved elastic fiber as in Fig. 4. If we apply a force $F$ to the fiber as indicated in Fig. 4 it will elongate by a distance $u$ which for small $F$ is linearly related to $F$ via $k u=F$, where 


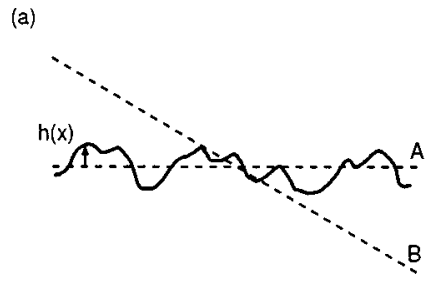

(b)

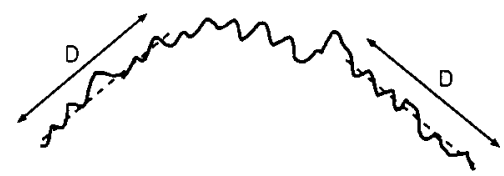

FIG. 8. (a) The reference plane (dashed line), from which the height $h(x)$ is measured, must be chosen so that not only $\langle h\rangle=0$ (which is satisfied for both planes A and B), but also so that $\left\langle h^{2}\right\rangle$ is minimal (plane A). (b) The orientation of the reference plane (dashed line) depends on the position of the patch $D \times D$ on the rough substrate surface.

$$
k=C \frac{E R^{4}}{L^{3}},
$$

where $C$ is a number which depends on the exact shape of the fiber but which is of order $C \approx 10$. Now, the setae of the Tokay gecko (for which experimental data on toe-adhesion is available) is about $L \approx 110 \mu \mathrm{m}$ long and has a radius $R \approx 2$ $\mu \mathrm{m}$. The setae are made from (mainly) keratin, ${ }^{14}$ with an elastic modulus $E \approx 4 \mathrm{GPa}$. Thus we calculate $k \approx 6 \mathrm{~N} / \mathrm{m}$. Using the maximum (measured) setae pull-off force ${ }^{2} f$ $\approx 200 \mu \mathrm{N}$ gives $\sigma_{0}=f / k=33 \mu \mathrm{m}$ and the elastic energy [see Eq. (6) $] \epsilon_{\mathrm{el}}=k \sigma_{0}^{2} / 2 \approx 3 \times 10^{-9} \mathrm{~J}$. Since the density of setae in the gecko toe-pad is about $10^{4} \mathrm{~mm}^{-2}$ we get from (10) for a flat substrate $(G=1), \quad \gamma_{\mathrm{eff}} \approx 30 \mathrm{~J} / \mathrm{m}^{2}$ or $2 \mathrm{eV} / \AA^{2}$ in good agreement with the value deduced from the measured toe pull-off force using the JKR theory (see Sec. II).

Let us now address the following question: Why does the lizard adhesive system consist of a hierarchy of fibers, namely "long thick" fibers, followed by "short thin" fibers, followed by molecular chain "fibers;" see Fig. 2. Why not just a dense layer of "thin" fibers? We believe that the origin of the hierarchical structure of the adhesive microstructure is related to the fractal nature of all real surfaces, and to the van der Waals interaction between the fibers which makes a dense array of thin fibers unstable against "condensation" into a rigid compact sheet structure.

Naturally occurring surfaces (e.g., a stone wall) have surface roughness on all length scales, from macroscopic to atomistic. Adhesion between two bodies is only possible if the surfaces are able to deform (elastically or plastically) to make direct (atomic) contact at a non-negligible fraction of the nominal contact area. For "hard" solids this is nearly impossible and as a result adhesion is usually negligible between hard rough surfaces. ${ }^{15}$. The skin of the gecko toe-pad is able to deform and follow the substrate roughness profile on length scales much longer than the thickness $d \approx 100 \mu \mathrm{m}$ of the elastic keratin film, say, beyond $\sim 1000 \mu \mathrm{m}$. At shorter length scales the keratin film, because of its high elastic modulus (of order $4 \mathrm{GPa}$ ), can be considered as rigid and

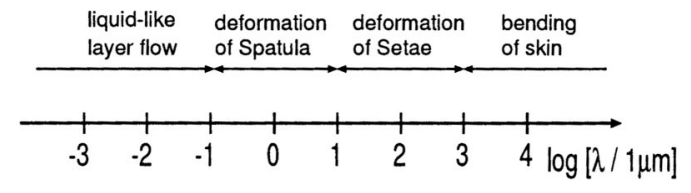

FIG. 9. A different part of the pad adhesive system is involved at different length scales $\lambda$.

flat. Elastic deformation of the pad surface on length scales shorter than $\sim 1000 \mu \mathrm{m}$ involves the compliant setae fiber array system (see above), with fibers of thickness $\sim 4 \mu \mathrm{m}$. If the surface roughness amplitude $\sigma$, measured over a patch $D \times D$ with $D \approx 1000 \mu \mathrm{m}$, is smaller than the adhesion length $\sigma_{0}$ (see Sec. IV and Fig. 7), then the fiber array system is able to deform (without storing in it a lot of elastic energy) to follow the surface roughness in the wavelength range $10<\lambda$ $<1000 \mu \mathrm{m}$. However, if the setae fiber tips would be blunt and compact they would not be able to penetrate into surface "cavities" with a diameter less than a few $\mu \mathrm{m}$. Thus, negligible atomic contact would occur between the surfaces, and the adhesion would be negligible. For this reason, at the tip of each long (thick) fiber occurs an array of $\sim 1000$ thinner fibers (diameter of order $\sim 0.1 \mu \mathrm{m}$ ). These fibers are able to penetrate into surface roughness cavities down to length scales of a few tenths of a micrometer. However, if the thin fibers would have blunt and compact tips made from the same "hard" keratin as the rest of the fiber, then one would still obtain a very small adhesion, since a lot of elastic energy would be necessary to deform the surfaces of the thin fibers to make atomic contact with the substrate. I therefore suggest that the top of the thin fibers are covered by a soft compliant layer, e.g., a liquidlike (high mobility) layer of polymer chains grafted to the tip of the thin fibers, which may be derived from the keratin molecules of the thin fibers (e.g., a natural "degradation product" of keratin). This liquidlike layer, if thick enough, would be able to adjust to the substrate roughness profile over lateral distances below $\sim 0.1 \mu \mathrm{m}$. Such a high-mobility grafted monolayer film may show up in sliding friction experiments as a velocity-dependent kinetic friction force, which increases monotonically with increasing sliding velocity, ${ }^{16}$ as observed in model experiments by Israelachvili et al. ${ }^{17}$ In this picture, the hierarchic nature of the pad surface morphology reflects the fact that all natural surfaces (and most engineering surfaces as well) have surface roughness on many different length scales; see Fig. 9.

In light of the discussion above one may ask why a gecko toe-pad has not just a single layer of very thin and long fibers, which could be easily deformed without storing up a large elastic energy. I believe that there are two explanations to this question. First, very long thin fibers may be more prone to wear than the more rigid hierarchic structure. Second, a dense array of very thin and long fibers would be unstable against "condensation" (because of the fiber-fiber van der Waals interaction) into a compact thin film with a high effective elastic modulus (see Appendix C). This fact is actually well known at the molecular level: hydrocarbon chains grafted to a solid substrate often form a compact structure consisting of parallel molecular chains with their axis tilted relative to the substrate normal; this configuration 
maximize the van der Waals binding energy between the chain molecules. ${ }^{18}$ To tilt or bend a molecule requires an elastic bending energy (and result in a reduction in the entropy), but if the chains are long enough, the bending energy is more than compensated by the gain of chain-chain binding energy.

\section{SUMMARY}

I have studied adhesion relevant to biological systems, e.g., flies, crickets and lizards, where the adhesive microstructures consist of arrays of thin fibers. The effective elastic modulus of the fiber arrays can be very small which is of fundamental importance for adhesion on smooth and rough substrates. I have shown how the adhesion depends on the substrate roughness amplitude and applied the theoretical results to the adhesion pads of lizards.

The construction of man-made adhesives based on fiber array systems may be an attractive alternative to the usual adhesives based on thin polymer films. In particular, while pressure sensitive adhesives (polymer films) are easily contaminated, e.g., by dust, sand particles, or liquids, fiber array systems may exhibit large contact angles for liquids (i.e., good nonwetting properties), and exhibit self-cleaning properties, as observed for leafs from many plants (the so called lotus-leaf effect), ${ }^{20}$ and which result from arrays of wax microstructures on the surfaces of the leafs, with typical size order of a few $\mu \mathrm{m}$.

\section{ACKNOWLEDGMENTS}

The author thanks S. Gorb for useful information and K. Autumn for a preprint. Financial support from BMBF (German-Israeli Project Cooperation: "Novel Tribological Strategies from the Nano- to Meso-Scales") is gratefully acknowledged.

\section{APPENDIX A: FIBER DEFORMATION ENERGY}

Consider a fiber which, in its undeformed state (i.e., in the absence of an applied force), has a curved shape; see Fig. 4. Let $x$ be a coordinate along the fiber and let $r_{0}(x)$ be the radius of curvature of the fiber (in its undeformed state) at point $x$. If $r(x)$ denote the radius of curvature at point $x$ after an external force has been applied, then the elastic bending energy, ${ }^{19}$

$$
E_{\text {fiber }}=\frac{1}{2} E I \int d x\left(\frac{1}{r}-\frac{1}{r_{0}}\right)^{2},
$$

where $I$ is the moment of inertia which for a fiber with circular cross-section (radius $R$ ) is given by $I=\pi R^{4} / 4$. If the original fiber is given by the equation $u(x)=u_{0}(x / L)^{2}$, where $L$ is the length of the fiber and if the force $F$ displaces the free end of the fiber a distance $u_{1}$ to $u_{0}+u_{1}$, then $1 / r_{0}$ $\approx 2 u_{0} / L^{2}$ and $1 / r \approx 2\left(u_{0}+u_{1}\right) / L^{2}$ so that

$$
E_{\text {fiber }} \approx \frac{1}{2} E I L\left(\frac{2 u_{1}}{L^{2}}\right)^{2}=\frac{\pi}{2} \frac{R^{4}}{L^{3}} E u_{1}^{2} .
$$

This must equal the spring energy $k u_{1}^{2} / 2$ which gives the effective fiber spring constant,

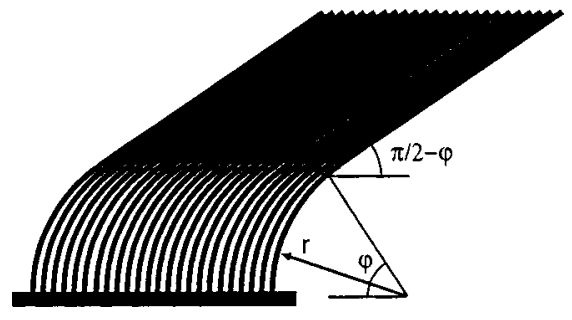

FIG. 10. The van der Waals interaction between a regular array of long thin fibers induces a transition into a compact dense layer which minimizes the total energy.

$$
k=\frac{C E R^{4}}{L^{3}},
$$

where $C$ is a number which depends somewhat on the shape of the fiber, but which typically is of order 10 .

We note that this effective bending spring is much softer than the spring due to elongation of the fiber. The latter is easy to calculate using the stress-strain relation $\sigma=E \epsilon$ and using $F=\pi R^{2} \sigma$ and $L \epsilon=u$ gives the force-displacement relation $F=k^{\prime} u$ where $k^{\prime}=\pi R^{2} E / L$. Thus the ratio $k / k^{\prime}$ $=(C / \pi)(R / L)^{2} \approx 10^{-3}$. Thus we can neglect the spring $k^{\prime}$ and assume that the fiber has only bending elasticity.

\section{APPENDIX B: PLATE DEFORMATION ENERGY}

Let $u(\mathbf{x})$ denote the vertical displacement field of a thin plate which originally (in the undeformed state) occupies the $x y$-plane. The elastic energy stored in the plate is given by ${ }^{19}$

$$
E_{\text {plate }}=\frac{E d^{3}}{24\left(1-\nu^{2}\right)} \int d^{2} x\left[\left(\nabla^{2} u\right)^{2}-2(1-\nu)\left|u_{i j}\right|\right],
$$

where the determinant

$$
\left|u_{i j}\right|=\frac{\partial^{2} u}{\partial x^{2}} \frac{\partial^{2} u}{\partial y^{2}}-\left(\frac{\partial^{2} u}{\partial x \partial y}\right)^{2} .
$$

Now, consider a deformation of the plate over an area $\sim D^{2}$ with the displacement $\sim u_{1}$. In this case $\nabla^{2} u \sim u_{1} / D^{2}$ and similar for $\left|u_{i j}\right|$ so that

$$
E_{\text {plate }} \approx E d^{3} D^{2} u_{1}^{2} / D^{4}=E d^{3} u_{1}^{2} / D^{2} .
$$

In general, depending on the shape of the "indentation," we may write

$$
E_{\text {plate }}=Q E d^{3} u_{1}^{2} / D^{2},
$$

where $Q$ is a number of order unity.

\section{APPENDIX C: FIBER CONDENSATION ENERGY}

Consider $N$ thin (radius $R$ ) but long (length $L$ ) elastic fibers bound with their axis perpendicularly to a rigid sheet. If the ratio $L / R$ is large enough, the fibers will deform (see Fig. 10) to form a compact layer of tilted fibers. Let us calculate the energy $U$ for the fiber system. Assume that the fibers have a constant curvature $r$ in the bended region. The elastic energy stored in the fibers will then be

$$
U_{\mathrm{el}}=N E I \varphi / 2 r
$$


where the angle $\varphi$ is defined in Fig. 10 and is given by $\varphi$ $=\cos ^{-1}\left(n R^{2} 2 \sqrt{ } 3\right)$, where $n$ is the number of fibers per unit area. Note that $0<\varphi<\pi / 2$. We assume that the fiber bind to each other (via the van der Waals interaction) over a length $L^{\prime}=L-\varphi r$. If $\epsilon^{\prime}$ denote the fiber-fiber binding energy per unit length, then the total energy,

$$
U=N E I \varphi / 2 r-3 N \epsilon^{\prime}(L-\varphi r) \equiv A / r-B+C r .
$$

Minimizing $U(r)$ with respect to the radius of curvature $r$ gives $r=(A / C)^{1 / 2}$. Substituting this in $U$ gives

$$
U=2(A C)^{1 / 2}-B=N\left[\left(6 E I \epsilon^{\prime}\right)^{1 / 2} \varphi-3 \epsilon^{\prime} L\right] .
$$

When the fibers are straight we can neglect the van der Waals interaction between them so that the undeformed fiber state has zero energy. Thus, fiber condensation will occur if the total energy $U$ given by $(\mathrm{C} 1)$ is negative. Hence, the condition $U=0$ corresponds to the case where the two states have equal energy. This gives

$$
\frac{L}{R}=\left(\frac{\pi}{6} \frac{E R^{2}}{\epsilon^{\prime}}\right)^{1 / 2} \varphi .
$$

When $L / R$ is larger than determined from $(\mathrm{C} 2)$, then the fiber array will condense into the densely packed state shown in Fig. 10. For two parallel fibers, separated by a small distance $b^{*}$, of order a few $\AA$, one can calculate the van der Waals fiber-fiber interaction energy (per unit length) ${ }^{21}$

$$
\epsilon^{\prime} \approx \frac{\pi}{2} \Delta \gamma\left(R b^{*}\right)^{1 / 2}
$$

Substituting this in (C2) gives

$$
\frac{L}{R} \approx\left(\frac{E R^{3 / 2}}{3 \Delta \gamma b^{* 1 / 2}}\right)^{1 / 2} \varphi
$$

For $R=0.1 \mu \mathrm{m}$ thick keratin fibers (typical of spatula) with $E \approx 4 \mathrm{GPa}$ and $b^{*} \approx 2 \AA$ (direct fiber-fiber contact), we get $L / R \approx 300 \varphi$. Since typically $\varphi \sim 1$, this shows that the array of thin fibers (spatula) cannot be longer than about $30 \mu \mathrm{m}$ without condensing into a compact structure. The actual length of the spatula $(\approx 20 \mu \mathrm{m})$ is just slightly shorter than this theoretical limit, which illustrates how nature, via the principle of natural selection, has optimized the lizard adhesive system.

${ }^{1}$ M. Scherge and S. Gorb, Biological Micro- and Nano-Tribology (Springer-Verlag, Berlin, 2001).

${ }^{2}$ K. Autumn, M. Sitti, Y. A. Liang et al., Proc. Natl. Acad. Sci. U.S.A. 99, 12252 (2002); K. Autumn, Y. A. Liang, S. T. Hsieh, W. Zesch, W. P. Chan, T. W. Kenny, R. Fearing, and R. J. Full, Nature (London) 405, 681 (2000).

${ }^{3}$ B. N. J. Persson, Phys. Rev. Lett. 81, 3439 (1998); J. Chem. Phys. 110, 9713 (1999)

${ }^{4}$ J. G. Williams, Adv. Polym. Sci. 27, 67 (1978)

${ }^{5}$ K. N. G. Fuller and D. Tabor, Proc. R. Soc. London, Ser. A 345, 327 (1975).

${ }^{6}$ G. A. D. Briggs and B. J. Briscoe, J. Phys. D 10, 2453 (1977).

${ }^{7}$ B. N. J. Persson, Eur. Phys. J. E 8, 385 (2002); Phys. Rev. Lett. 89, 245502 (2002).

${ }^{8}$ D. J. Irschick, C. C. Austin, K. Petren, R. Fisher, J. B. Losos, and O. Ellers, Biological J. Linnaen Soc. 59, 21 (1996).

${ }^{9} \mathrm{~K}$. Autumn and A. Peattie (unpublished).

${ }^{10}$ S. Sponberg, W. Hansen, A. Peattie, and K. Autumn, Int. Comp. Bio. 39, 395 (2002).

${ }^{11}$ K. L. Johnson, K. Kendall, and A. D. Roberts, Proc. R. Soc. London, Ser. A 324, 301 (1971).

${ }^{12}$ D. T. V. Pawluk and R. D. Howe, ASME J. Biomech. Eng. 121, 605 (1999).

${ }^{13}$ The elastic modulus of cuticle has been measured by different authors: 13 GPa for the locust and beetle cuticle [R. F. Ker, Ph.D. thesis, University of Oxford (1977)]; $10 \mathrm{GPa}$ for the locust cuticle [M. Jensen and T. WeisFogh, Philos. Transact. 245, B721 (1977); 0.3 GPa for the insect exoskeletons [H. P. Hepburn and I. Joffe, in The Insect Integument, edited by H. R. Hepburn (Elsevier, Amsterdam, 1976), pp. 107-235]; 0.3 GPa for the tarsal claw system in the beetle Pachnoda Marginata [Z. Dai, S. N. Gorb, and U. Schwarz, J. Exp. Biol. 205, 2479 (2002)].

${ }^{14}$ Keratin is a group of fibrous proteins occurring in hair, feathers, hooves and horns.

${ }^{15}$ B. N. J. Persson, Sliding Friction: Physical Principles and Applications, 2nd ed. (Springer, Heidelberg, 2000).

${ }^{16}$ The physical reason for this is simple: At low sliding velocity the molecules in the liquidlike grafted film can "jump" out of their substrate pinning wells by thermal fluctuations, giving a small friction force. At high sliding velocity the molecules are pulled out of the pinning wells by the sliding motion (i.e., there is not enough time for them to get thermally depinned), giving rise to a much higher friction force. Recent experiments have indeed shown that the friction force between a gecko setae and a substrate increases monotonically with increasing sliding velocity $[\mathrm{K}$. Autumn (private communication); see also Ref. 10].

${ }^{17}$ H. Yoshizawa, Y.-L. Chen, and J. Israelachvili, J. Phys. Chem. 97, 4128 (1993).

${ }^{18}$ M. Salmeron, Tribol. Lett. 10, 69 (2001).

${ }^{19}$ L. D. Landau and E. M. Lifshitz, Theory of Elasticity (Pergamon, London, 1959).

${ }^{20}$ C. Neinhuis and W. Barthlott, Ann. Bot. (London) 79, 667 (1997).

${ }^{21}$ J. N. Israelachvili, Intermolecular and Surface Forces (Academic, London, 1995). 